\title{
Starvation Due To Neglect: A Canine Case Series
}

\section{Adam W. Stern*}

Veterinary Diagnostic Laboratory, University of Illinois, Urbana, USA

\begin{abstract}
Identification of emaciated animals is a straight-forward. Occasionally, the cause of the emaciated condition is not natural, and neglect is suspected. In this report, several cases of starvation due to neglect are described and the diagnosis of neglect due to starvation will be discussed.
\end{abstract}

Keywords: Neglect; Starvation; Dog; Forensic science

\section{Introduction}

Both clinical veterinarians and veterinary pathologists are often presented with a severely emaciated animal and in a majority of cases the animal's body condition is due to natural disease. Unfortunately in some cases there is no underlying natural disease and the animal's condition is human induced (animal abuse, specifically neglect). In veterinary medicine neglect is separated into four man categories: failure to proved adequate food, water, shelter, and veterinary care [1]. Recognition of an emaciated animal is relatively straightforward and oftentimes underlying natural disease is readily diagnosed. Body condition scoring provides an indication of nutrition in the living animal and use of one of the several available body condition score systems is mandatory (i.e., Purina 9 step body condition score). If the animal is no longer living, it is generally accepted to describe an animal's nutritional condition as obsess, adequate, and emaciated. If the animal died or was euthanized, a complete post mortem examination should be performed. In cases of an animal in an emaciated condition, ancillary diagnostic tests including radiographs, blood work, fecal examination, and gastrointestinal biopsy may be performed. As a veterinarian it is our duty to determine if the results of the tests performed and subsequent diagnosis could have resulted in this animal's condition. If the cases findings are inconclusive or do not support natural disease as a cause for the emaciated condition of the animal, neglect should be considered as a possible differential.

\section{Case 1}

An intact female, adult, $15 \mathrm{~kg}$ pit bull-type dog was relinquished to animal control. The animal had a body condition score of 2/9. Neglect

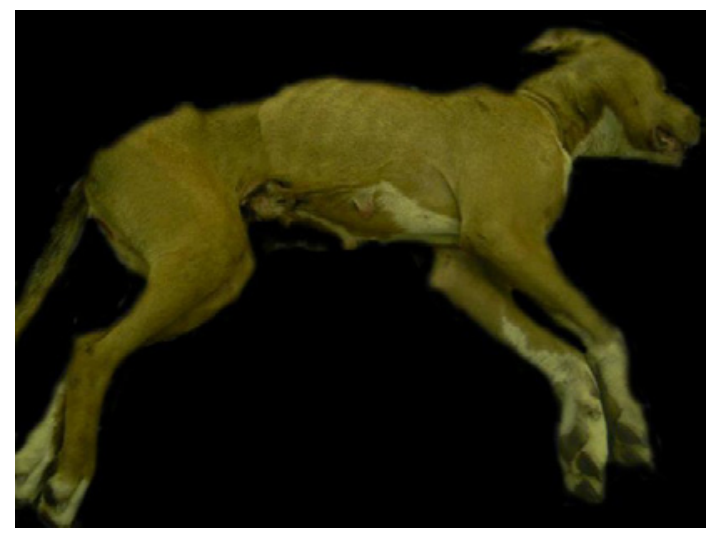

Figure 1: Note the rib and pronounced prominences of the pelvis. specifically starvation was suspected and the dog was humanely euthanized.

At necropsy examination the animal had easily visible and palpable ribs, vertebral prominences, and long bone prominences (Figure 1). There was lack of subcutaneous and mesenteric adipose tissue. Epicardial adipose was scant. Within the gastrointestinal tracts is a scant amount of mucoid yellow material. There is a combination of dirt and foreign material (Styrofoam). Toxocara spp. parasites were within the small intestine. Long bones are sagittally sectioned and there was deposition of yellow and gelatinous material within the diaphyses. The liver was smaller than expected and did not extend beyond the costochondral arch. Organ weights were recorded and the weights of the liver and kidney were small compared to the expected body weight, organ ratio.

Histologic examination revealed muscle atrophy and serous atrophy of bone marrow and there was no evidence of systemic disease. Based on these post mortem findings and case investigation the cause of disease was starvation due to neglect.

\section{Case 2}

An intact male, adult, $16.8 \mathrm{~kg}$ pit bull-type dog was relinquished to animal control for suspected neglect. The animal had a body condition score of $2 / 9$. Due to the poor prognosis the animal was humanely euthanized.

At necropsy examination the animal had easily visible and palpable ribs, vertebral prominences, and long bone prominences. There was lack of subcutaneous and mesenteric adipose tissue. Epicardial adipose was scant. Within the gastrointestinal tracts, there is a moderate amount of dirt. There is a combination of a marked amount of dirt, straw and Styrofoam within the stomach. Toxocara spp. parasites were within the small intestine. Long bones are sagittally sectioned and there was deposition of yellow and gelatinous material within the diaphyses. The liver was smaller than expected and did not extend beyond the costochondral arch. Organ weights were recorded and the weights of

*Corresponding author: Adam W. Stern, DVM, CMI-IV, CFC, Diplomate ACVP Veterinary Diagnostic Laboratory, University of Illinois, Urbana, IL 61802-217-3331620, USA, E-mail: awstern@Illinois.edu

Received December 31, 2012; Accepted January 30, 2013; Published February 01, 2013

Citation: Stern AW (2013) Starvation Due To Neglect: A Canine Case Series. J Veterinar Sci Technol 4: 131. doi:10.4172/2157-7579.1000131

Copyright: (C) 2013 Stern AW. This is an open-access article distributed under the terms of the Creative Commons Attribution License, which permits unrestricted use, distribution, and reproduction in any medium, provided the original author and source are credited. 
the liver and kidney were small compared to the expected body weight, organ ratio.

Histologic examination revealed muscle atrophy and serous atrophy of bone marrow and there was no evidence of systemic disease. Based on these post mortem findings and case investigation the cause of disease was starvation due to neglect.

\section{Case 3}

An intact male, $23.6 \mathrm{~kg}$ mixed breed dog was found deceased within an outdoor kennel with no food and water. The house where the dog was found was abandoned. Animal control officers submitted the animal for post mortem examination.

At necropsy examination the animal was in poor nutritional condition (emaciated) having easily visible and palpable ribs, vertebral prominences, and long bone prominences. There is lack of subcutaneous and mesenteric adipose tissue. Epicardial fat is absent. The stomach contained a large amount of poorly chewed dog kibble. Within the remainder of the gastrointestinal tract is a scant amount of mucoid yellow material. Long bones are sagittally sectioned and there is deposition of yellow and gelatinous material within the diaphyses. The liver was smaller than expected and did not extend beyond the costochondral arch. Organ weights were recorded and the weights of the liver and kidney were small compared to the expected body weight, organ ratio.

Histologic examination revealed muscle atrophy and serous atrophy of bone marrow. There was no evidence of systemic disease within the animal. The cause of death was suspected to be due to refeeding syndrome and investigation identified a neighbor who said they had recently fed this dog. Based on these post mortem findings and case investigation the immediate cause of death was refeeding syndrome and the proximate cause of death was starvation due to neglect.

\section{Discussion}

The findings in these cases are consistent with starvation as a result of neglect. In such circumstances, the correct diagnosis of starvation due to neglect can easily be missed if a thorough examination was not performed. These cases highlight how the differential diagnosis of animal abuse should be considered when presented with an emaciated animal. It should be noted that dogs are more likely that cat to be victims of starvation [2].

From a postmortem point of view lack of adipose tissue stores specifically from the subcutaneous regions and mesentery are often encountered often in conjunction with skeletal muscle atrophy which begins to occur after 24 hours of starvation [1]. Histologically both type 2 skeletal muscle fibers are preferentially affected [3]. With time there will be loss of perirenal, epicardial and bone marrow adipose tissue and there can be replacement of fat by yellow to tan gelatinous material particularly within the epicardial region and bone marrow (serous atrophy of fat). Quantitative assessment of the percent of fat in bone marrow can be performed and test results can be used in conjunction with other case findings to support a diagnosis of starvation or malnutrition [4].

Examination of the entire gastrointestinal tract both grossly and histologically is imperative. Animals that are starved typically have a gastrointestinal tract devoid of ingesta/digesta and contain a mild amount of yellow mucoid material. Gastric and intestinal foreign materials are not uncommonly observed within starved animals [1]. The gastrointestinal tract of starved animals is considerably less malodorous compared to a health and well-nourished animal [1].

The body weight and organ weights of the animal should be taken. In cases of starvation in human beings, anthropometrical data of starved people and organs weights are routinely compared to those of a reference population and necessary in the diagnosis of starvation [5]. Organ weights of the animal being examined should be compared to a reference population for that animal species (and breed). Comparison only to the animals' bodyweight is considered inadequate. In human beings, loss of approximately $35-50 \%$ of body weight may cause death, and loss of body weight mainly results from loss of adipose tissue, atrophy of internal organs, and atrophy of muscle [5].

During the thorough postmortem examination, natural disease will occasionally be diagnosed. It is the job of the veterinarian to determine whether the disease could have resulted in the condition of the animal or is it secondary to the emaciated condition or even an incidental finding. Although beyond the scope of this article, measurement of the volume and nutritional value of the food the animal is eating may also support the diagnosis of starvation [2].

In summary, these cases highlight some of the common findings observed in animals that are starved to neglect. Both gross and Histologic examination of deceased animals is imperative to rule in/ out natural disease that could have results in the animal's emaciated condition. The postmortem findings in conjunction with investigations performed by law enforcement will allow for the diagnosis of starvation due to neglect.

\section{References}

1. Munro R, Munro MCM (2008) Animal Abuse and Unlawful Killing: Forensic Veterinary Pathology. Saunders, Philadelphia, USA.

2. Sinclair L, Merck M, Lockwood R (2006) Forensic Investigation of Anima Cruelty: A Guide for Veterinary and Law Enforcement Professionals. Humane Society Press, Washington DC, USA.

3. Van Fleet JF, Valentine BA (2007) Muscle and tendon: Pathology of domestic animals. Saunders, Philadelphia, USA.

4. Meyerholtz KA, Wilson CR, Everson RJ, Hooser SB (2011) Quantitative assessment of the percent fat in domestic animal bone marrow. J Forensic Sci 56: 775-777

5. Madea B (2005) Death as a result of starvation. Forensic Pathology Reviews 2: 3-23. 